This report was prepared as an account of work sponsored by an agency of the United States Government. Neither the United States Government nor any agency thereof, nor any of their employees, makes any warranty, express or implied, or assumes any legal liability or responsibility for the accuracy, completeness, or usefulness of any information, apparatus, product, or process disclosed, or represents that its use would not infringe privately owned rights. Reference herein to any specific commercial product, process, or service by trade name, trademark, manufacturer, or otherwise does not necessarily constitute or imply its endorsement, recommendation, or favoring by the United States Government or any agency thereof. The viaws and opinions of authors expressed herein do not necessarily state or reflect those of the United States Government or any agency thereof.

\title{
RISK COMMUNICATION IN ENVIRONMENTAL RESTORATION PROGRAMS
}

J. A. Bradbury

Apri7 1993

Presented at the

The Risk Assessment

Paradigm After 10 Years

Apri1 5-8, 1993

Dayton, Ohio

Prepared for

the U.S. Department of Energy

under Contract DE-ACO6-76RLO 1830

Pacific Northwest Laboratory

Richland, Washington 99352 
The author advocates adoption of a convergence model in place of the traditional sourcereceiver model of communication for communicating with members of the public who have a stake in remediation of a nearby site. The source-receiver model conceives of communication as the transmission of a message from a risk management agency (sender) to a target audience of the public (receivers). The underlying theme is that the sender intends to change the perception of the receiver of either the issue or the sender of information. The theme may be appropriate for health campaigns which seek to change public behavior; however, the author draws on her experience at a DOE site undergoing remediation to illustrate why the convergence model is more appropriate in the context of cleanup. This alternative model focuses on the Latin derivation of communication as sharing or making common to many, i.e., as involving a relationship between participants who engage in a process of communication. The focus appears to be consistent with recently issued DOE policy that calls for involving the public in identifying issues and problems and in formulating and evaluating decision aiternatives in cleanup. By emphasizing context, process and participants, as opposed to senders and receivers, the model identifies key issues to address in facilitating consensus concerning the risks of cleanup. Similarities between the institutional context of DOE and DOD suggest that a convergence model may also prove to be an appropriate conceptual foundation for risk communication at contaminated DOD sites.

Key Words:

Risk communication

Environmental remediation

Public participation

Models of communication

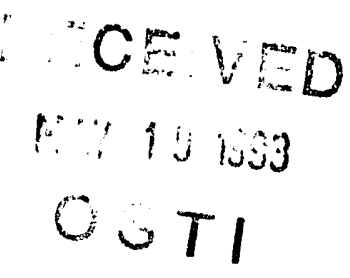




\section{Introduction}

At a national conference on risk communication held in 1986, William Ruckelshaus (1), former Administrator of the Environmental Protection Agency, emphasized:

My point is not to say whether a sharing of power to make risk management decisions is right or wrong; it is simply to state that it is a fact of life in the United States. We have decided in an unprecedented way, that the decision making responsibility involving risk issues must be shared with the American people . . . So the question before us is not whether there is going to be any sharing, whether we will have participatory democracy with regard to the management of risk, but how.

My topic today is risk communication in the context of sharing in risk decisions with members of the public who have a stake in remediation of a nearby contaminated site. First, I will briefly summarize and provide my personal perspective on developments occurring under the Environmental Restoration and Waste Management (EM) program of the Department of Energy (DOE). Subsequently, I will outline two models of communication--the linear model and the convergence model. The focus of the discussion is on the implications of each model for practice: which model provides the more appropriate basis for risk communication in the context of sharing in the management of risk? I argue that lack of clarity concerning the purpose of risk communication in different contexts and for different types of policy is contributing to continued confusion over how to structure the sharing process. 


\section{Experience from the Field}

Creighton (2) has recently pointed to changes that have occurred over the past few decades in public expectations concerning agency decision making. He argues that, in the post World War II era, expectations were limited to information, which was provided at the agency's discretion. Expectations shifted to procedural public involvement in the 1960s and 1970 s, while the 1980 s witnessed increasing calls for "meaningful" participation in agency decisions, i.e., a role in defining the problem and in determining the range of alternatives considered and the criteria used to evaluate those alternatives. Creighton has identified collaborative decision making as the expectation of the 1990 s.

Changed expectations, in combination with pervasive public distrust of government institutions (3), have had particular impact on the Department of Energy (DOE), where decision making was formerly cloaked in the secrecy afforded by a national security mission. In the post cold war era, the agency is now faced with the need to remediate over 9,000 sites in full compliance with a range of environmental regulations. The Decide-Announce-Defend approach of former years perforce is giving way to attempts to develop a new culture of openness and involvement of the various publics (stakeholders) ${ }^{1}$ in agency decision making.

As with any culture change, the transition will not occur overnight. The agency is likely to encounter disagreements, lack of understanding, and confusion over the extent and implications of this new approach to decision making. Individual staff may be expected to vary in their commitment to public participation; preferred approaches are likely to span the continuum from providing information, to providing a consultative role for the public, and ultimately to providing for public involvement in formulating and reaching decisions. 
Meanwhile, public participation activities are increasing at the many sites in the DOE complex. Within the past year, a newly established office of policy and program information in the Environmental Restoration and Waste Management (EM) program has provided for increased coordination among the sites and between headquarters and field offices. At the national level, two advisory groups meet and interact on a regular basis with program staft: the State and Tribal Government Working Group and the EM Advisory Committee for the Programmatic Environmental Impact Statement.

These activities, however, are unlikely per se to address what I consider to be the most critical need in meeting public expectations of the 1990 s and in increasing public trust in the decision-making process. In my view, this is the need to integrate public participation activities into the technical decision-making process specified under the Comprehensive Environmental Response, Compensation, and Liability Act (CERCLA). Significantly, although the CERCLA regulations provide for a more formal program in public participation than other environmental statutes do, DOE (or the applicable regulated entity) is not required to engage the public in early discussion of plans and alternatives, including discussion of risks. All that is required is availability of information in a nearby information repository and formal public comment on the agency's preferred cleanup alternative. The regulations therefore do little to facilitate a change in DOE's normal way of doing business, i.e. of making decisions, frequently classified as "purely technical," without benefit of early public input. Too often, public participation becomes an add-on to the real action: public participation activities consist of informing the public and/or seeking formal comments on decisions that, essentially, have already been reached. Such an approach is unlikely to meet public expectations in the 1990s or to engender public trust. 
The lack of integration between technical and public participation activities is especially marked in addressing and communicating about risk, which frequently symbolizes and serves as the lightning rod for disagreement between technical and lay communities on cleanup issues. An increasingly formalized approach to risk assessment underlies the entire RI/FS decision-making process, culminating in selection of a preferred cleanup alternative. Here, the lack of CERCLA requirements for early public input is reinforced by EPA policy which has consistently attempted to make a clear distinction between risk assessment and risk management. ${ }^{2}$ The theoretical premise underlying this policy assumes that risk is a completely objective, neutral entity. The unfortunate practical result of the policy is that it contributes to a continuation of a technocratic, rather than a participatory approach to decision making. This approach views risk communication as a process of providing technical risk information to the public--in effect, attempting to persuade the public to accept as the basis for action the technical risk assessments from which they are excluded.

The notion that risk is a completely objective, neutral entity has been challenged by writers who adhere to a social constructivist viewpoint (4-10). This viewpoint conceives of risk as a socially constructed attribute rather than a physical entity that exists independently of the humans who assess and experience its effects. Authors who adhere to this viewpoint emphasize the inherent uncertainties in risk assessment and the element of judgment that underlies both technical and lay assessments of risk. Accordingly, they advocate negotiation on value differences as the appropriate approach to programs and policies involving risk issues. Fiorino (11) summarizes the current situation as follows:

The risk community has focused its attention on the technical and economic aspects of policy making. Yet the challenge to effective risk management may not be so much the techrical or economic as political. By political, I mean the ways people 
view their relationship to institutions making collective decisions about environmental risk and their capacities for influencing those decisions. Yet the literature on environmental risk--whether it assumes the label of risk assessment, management, or communication--often ignores this aspect of risk problems and their solutions. ... We refine, polish, and perfect our formal models for determining acceptable levels of risk, despite evidence that the assumptions and methods bear little relationship to the lay public's conception of the problems. We test techniques for communicating risk information to the public, but conduct almost no research on mechanisms for the lay public to communicate with government officials and technical experts.

Several events that have occurred within the past few months may, in combination, provide greater support for early stakeholder input and foster collaborative decision making among DOE and its various stake holders. First, EM public participation policy, which was issued in the fall of 1992 , commits the program to provide opportunities for public participation in program planning, design, and implementation. The policy specifically promises to provide "opportunities for the public to assist DOE in identifying EM-related issues and problems and in formulating and evaluating decision alternatives." Second, the interim report of the Federal Facilities Environmental Restoration Dialogue Committee inclucies a recommendation that will impact all Federal agencies involved in remediation. ${ }^{3}$ The Committee recommends establishment of site-specific advisory boards composed of a representative cross-section of stakeholders and including senior staff of the agency subject to regulation. Third, the Defense Reauthorization Act for Fiscal Year 1993 requires DOE to submit a report to Congress on the effectiveness of existing advisory groups, the desirability of establishing new or replacement advisory groups, and methods for improving public 
participation in EM activities. DOE is already in the process of forming advisory groups of stakeholders at five large sites within the DOE complex.

Establishment of site-specific advisory boards may prove to be a critical development. Over time, such boards could provide for genuine dialogue, setting the stage for negotiation on contentious issues surrounding cleanup. Confusion in the social and communication sciences, however, has hindered development of a sound theoretical foundation for risk communication in such settings. The lack of conceptual clarity is particularly regrettable, given DOE's need for guidance in structuring participation during an era of fundamental culture change.

\section{Two Models of Cornmunication}

Everett Rogers, a leading scholar in the field of communication, has distinguished between two models of communication: the linear model and the convergence model. These theoretical distinctions have practical implications for risk communication in the context of remediation.

The Linear Model, shown overpage, portrays communication as the transmission of a message, via a channel (media), from a source (sender) to a receiver. Communication, here, is conceptualized as a one-way process, albeit with feedback loops that allow for a reaction, in which information is provided to someone. As emphasized by Rogers, information is viewed as c:ontext-free, to be "carried from a source to a receiver the way a bucket carries water" (12). The focus of the linear model is the effect of communication on the receiver-essentially the goal is persuasion; typically, as suggested recently by Renn (13), a risk management agency is viewed as the communicator and groups of the public are "target 
audiences." Important issues for research and practice include how to facilitate attitude change and how to promote consistency between attitudes and behavior in the intended receiver.

Renn has observed that the linear model continues to be the dominant model underlying much of the current discussion of risk communication. Covello, von Winterfeldt, and Slovic (14), for example, emphasize problems with the source, message, channel, and receiver that hinder the recipient's ability to receive (and act on) the message. This emphasis, implicitly incorporating the "blame the receiver" orientation of the linear model which has been criticized by Rogers, reinforces the viewpoint that the problem lies in lack of public understanding of the so-called "real" risks. More explicitly, Renn (15) asserts that "most authors" view the general purpose of risk communication as "aim[ing] at changing behavioral response."

In their discussion, however, these authors distinguish different types--and associated purposes--of risk communication. Covello, Von Winterfeldt, and Slovic provide a typology of four risk communication objectives:

- Information and education

- Behavior change and protective action

- Disaster warnings and emergency information

- Joint problem solving and conflict resolution

Renn similarly distinguishes three specific purposes of risk communication:

- To make sure that all receivers of the message are able and capable of understanding and decoding the meaning of messages sent to them

- To persuade the receivers of the message to change their attitudes or their behavior with respect to a specific cause or class of risk 
- To provide the conditions for a rational discourse on risk issues so that all parties can take part in an effective and democratic conflict-resolution process The authors' recognition that risk communication may be employed for different purposes, however, is not accompanied by recognition that the linear model provides an inadequate foundation for all of these purposes. It is questionable whether engaging in risk communication to "change [the public's] behavioral response" is consistent with Ruckelshaus's goal of "sharing. . . [and] participatory democracy with regard to the management of risks" or with DOE's new, participatory approach to decision making. Indeed, the social engineering orientation of the linear model is likely to prove counterproductive: risk bearers such as citizens affected by contamination and remediation of a nearby site are more likely to demand involvement in the decisions that affect them and to resent implications that their attitudes are in need of change.

The linear model may initially appear to provide an appropriate conceptual foundation for programs such as health campaigns that seek to persuade targeted groups of the need for changes in behavior. In this context, the data base is firmly established and programs that seek to change behavior seem justified: an accumulation of data indicates that changing public behavior (in relation to smoking, diet etc.) would result in improved health and longevity. Even here, however, the model fails to address the element of interpretation that enters into all human communication. The need for a different model on which to base policy is more pronounced in tne context of remedial programs. Needed data may be lacking and uncertainty is a key aspect of the decision problem. Moreover, implementing an effective clean-up remedy that is acceptable to stakeholders calls for joint problem solving and conflict resolution rather than persuasion. 
The need for a different model is highlighted by contradictions discernable in the National Research Council 1989 publication, Improving Risk Communication. The authors emphasize that risk communication is a two-way, interactive process--a "form of democratic dialogue," rather than a persuasive attempt to "get one's message across" (16). However, continued reliance on the terminology of the linear model for risk communication undermines their attempt to provide an interactional orientation. The implicitly persuasive orientation of the linear model provides a foundation that is in fundamental contradiction to an explicit emphasis on communication as a mutual process. The authors acknowledge the lack of "complete knowledge" that forms the basis for risk assessment, the difficulty of separating the underlying values of the risk assessor, and the factors that affect human, including scientific judgment. However, they fail to address this key issue of the inherent uncertainty and valueembedded nature of risk assessment, by implicitly adhering to a concept of risk as a physical attribute of hazardous technologies rather than a social constrliction that is affected by the social and cultural context. While advocating democratic dialogue on risk, the authors' frequent use of the tern "risk messages" endorses a view of communication as the transmission of context-free information. This emphasis on what is transmitted, rather than interpretation of the transmission and the transaction between groups who adhere to different values, reinforces the orientation toward communication as persuasion. Consequently, the authors fail to provide an adequate basis for structuring risk communication as a more participatory process.

The Convergence Model, in contrast, draws on the Latin derivation of communication: sharing, making common to many, or giving to another as a partaker. As Rogers and Rogers have emphasized, "Sharing implies a relationship ... that two or more people do something together, not that one individual does something to another .... [it] is not simply 
a matter of action and reaction--it is a transactional exchange between two or more individuals" (17).

In the convergence model, shown overpage, communication is a process in which participants are mutual communicators rather than senders and receivers. As indicated by the phrase "and then," participants bring to the process their own cultural frame of reference-their different experiences, values, and organizational background. The written or verbal symbols, which are the physical aspects of communication, may have a different meaning to each. In the communication process, participants share and create information, either diverging or converging on a common meaning or understanding. This sharing, transactional process is inherently a dialogue which takes place over a period of time. It is important to note that convergence on meaning does not necessarily mean agreement and the elimination of conflict.

Adopting a convergence model, however, shifts the linear model focus on the effect of the transmission on a recipient to a focus on the mutual nature of the communication process. The model also shifts the focus from differences between expert and laypersons to differences in expertise among all stakeholders, indicating different contributions that various parties provide in the communication process. Issues for research and practice include identification of these different sources of expertise, differences between frames of reference that hinder communication, and how and when to include risk bearers in the decision-making process. 


\section{Selecting an Appropriate Model}

The convergence model provides a more appropriate foundation than the linear model for risk communication in the context of dialogue among stakeholders concerning site remediation. Key, relevant features of the model are: (1) the focus on risk communication as a transaction provess; (2) justification for early stakeholder input; (3) the long-term, interactional nature of the process; and (4) consistency with relevant theoretical developments in the social sciences.

Risk Communication as a Transaction Process. The literature on public perception of risk has illuminated ways in which laypersons' assessments of risk differ from those of the technical risk assessor. There has been less widespread recognition, however, that technical judgments also represent perceptions. ${ }^{4}$ In particular, there has been less attention paid to the implications for policy of cultural differences among stakeholders in terms of perspectives and in acquiring and using knowledge (19). The convergence model points to the cultural filters that all participants bring to the communication process and highlights the need to take public concerns and knowledge seriously. It emphasizes the importance of communication as a transaction between participants with different frames of reference. The issues to address are not the effect of communication on the receiver and on how the technical expert can convey his message more effectively, i.e., convince the public, as in the linear model. Rather the issues are the barriers to communication on the part of all participants and the best way to structure a constructive dialogue among them.

Need for Early Stakeholder Input. By highlighting cultural differences between participants and the context in which communication occurs, the convergence model 
indicates the importance of communicating with rather than to stakeholders in the early stages of the risk assessment process. The level of acceptable risk to a community may depend on a variety of factors, including intended use of the site after remediation and the human health and environmental issues of most concern to those involved. Value-laden factors like these, which underlie many of the decisions that guide the conduct of risk assessment, confirm the fundamental impossibility of neatly distinguishing between the facts of risk assessment and the values of risk management. Establishing an early mechanism for communication will facilitate identification of value differences between risk assessors and other stakeholders, bringing risk bearers into the process to provide early guidance on their values. This guidance is required throughout, not simply after the conduct of the risk assessment.

The ongoing nature of communication. In the convergence model, communication is an iterative, long-term process rather than a single act of transmission. The model underscores the interactional nature of constructing common meanings. It also points to the importance of building a relationship as the basis on which a civilized debate can proceed.

Consistency with Theoretical Developments in the Social Sciences. Perhaps most significantly, the convergence model is consistent with two related trends in social thought that, in combination, provide a sound basis for designing policy on issues involving risk. First, the model provides a conceptual basis for communication as dialogue, consistent with the insights of Ravetz (20). Ravetz explicitly addresses the value-embedded nature of risk assessment in recommending a policy approach that is directly applicable to remediation policies and programs. He differentiates between types of policy problems according to location on two dimensions: a factual and a valuative dimension. A traditional, technical approach is suited to problems where a substantial body of data exists and value disputes 
among stakeholders are lacking. However, where the data base is not well established and value disputes arise, Ravetz recommends dialogue among stakeholders as a more appropriate approach to policy resolution.

Second, the convergence model supports an approach to policy that recognizes the limits of analysis as the basis for consensual social action. Habermas (21) has been a leacing contributor to the argument that stakeholders use different forms of rationality in evaluating proposed social actions. Forms of rationality include subjective and social dimensions in addition to the objective, analytic dimension provided by technical risk assessment. Issues such as trust and legitimacy of the decision process are key components of the subjective and social forms of rationality, respectively. The value of the convergence model is that the emphasis on establishing dialogue over a long period of time explicitly provides for the development of consensus based on ull forms of rationality.

\section{Conclusion}

The convergence model provides a conceptual foundation consistent both with theory and with the trend toward collaborative problem solving that is evident in the field. A key feature that demonstrates this consistency is the emphasis on communication as a transactional exchange between two or more individuals that involves developing common meanings and implies building a relationship. Communicating with rather than to the public is more likely to meet public expectations in the $1990 \mathrm{~s}$. 


\section{Notes}

1. The terms public and stakeholder are used interchangeably throughout to include the range of individuals and groups that are interested in or potentially affected by a policy or project, e.g., regulators and other Federal, State, Tribal, or local agencies; civic groups; national and regional interest groups; and the general public.

2. These distinctions, endorsed by EPA in 1984 (Risk Assessment and Management: Framework for Decision Making), were relied on to develop risk assessment guidelines (51 Federal Register, 33992-34054, September 24, 1986). The most recent statement confirming EPA's position is the Habicht memo, Guidance on Risk Characterization for Risk Managers and Risk Assessors, February 26, 1992.

3. The committee, which was established by the Environmental Protection Agency (EPA), is composed of 40 representatives of Federal, Tribal, and State governments and associations, and local and national environmental, community and labor organizations. The goal of the committee is to develop policy recommendations aimed at improving the decision-making process to ensure that clean-up decisions reflect the priorities and concerns of all stakeholders.

4. As noted by Fischhoff, Slovic, and Lichtenstein: "Although there are actual risks, nobody knows what they are. All that anyone does know about risks can be classified as perceptions" (16). 


\section{References}

1. William J. Ruckelshaus, 1986. "Overview of the Problem. Communicating about Risk." In Risk Communication: Proceedings of the National Conference on Risk Communication held in Washington, D.C., January 1986, eds. J.C. Davies, V.T. Covello, and F.W. Allen. Washington, D.C.: Conservation Foundation, p.5.

2. James L. Creighton, 1992. Welcoming Speech. International Association of Public Participation Practitioners Conference held in Portland, Oregon, September 1992.

3. Draft Final Report, 1992. Secretary of Energy Advisory Board on Radioactive Waste Management.

4. Jerome R. Ravetz, 1987. "Usable Knowledge, Usable Ignorance: Incomplete Science with Policy Implications," Knowledge, 9: 87-116.

5. Mary Douglas and Aaron Wildavsky, 1982. Risk and Culture. Berkeley: University of California Press.

6. Steve Rayner, 1987. "Risk and Relativism in Science for Policy." In The Social and Cultural Construction of Risk: Essays on Risk Selection and Perception. eds. B.B. Johnson and V.T. Covello, Boston: Reidel Publishing Company.

7. Sheldon Krimsky and Amos Plough, 1988. Environmental Hazards:

Communicating Risks as a Social Process. Dover, Massachusetts: Auburn House.

8. Harry J. Otway and Kerry Thomas, 1982. "Reflections on Risk Perception and Policy." Risk Analysis 2:69-82.

9. Judith A. Bradbury, 1989. "The Policy Implications of Differing Concepts of Risk." Science, Technology, and Human Values 14:380-399.

10. Center for Technology, Policy, and Industrial Development, 1991. Monitoring the Community for Exposure and Disease: Scientific, Legal, and Ethical Implications. Cambridge, Mas sachusetts: Massachusetts Institute of Technology.

11. Daniel J. Fiorino, 1989. "Environmental Risk and Democratic Process: A critical Review." Columbia Journal of Environmental Law, 14:501-547, p.503. p. 197.

12. Everett M. Rogers, 1986. Communication Technology. New York: Free Press,

13. Ortwin Renn, 1992. "Risk Communication: Toward a Rational Discourse with the Public." Journal of Hazardous Materials, 20:465-519, p.467. 
14. Vincent T. Covello, Detlof von Winterfeldt, and Paul Slovic, 1987.

"Communicating Scientific Information about Health and Environmental Risks." In Risk Communication: Proceedings of the National Conference on Risk Communication held in Washington, D.C.,January 1986, eds. J.C. Davies, V.T. Covello, and F.W. Allen, Washington, D.C.: Conservation Foundation.

15. Renn, loc. cit.

16. National Research Council, Committee on Risk Perception and Communication, 1989. Improving Risk Communication. Washington, D.C.: National Academy Press, pp. 2021.

17. Everett M. Rogers and Rekha Agawarla-Rogers, 1976. Cornmunication in Organizations. New York: Free Press, p.18.

18. Baruch Fischhoff, Sarah Lichtenstein, and Paul Slovic, 1983. "The Public vs. the 'Experts.'" In The Analysis of Actual vs. Perceived Risks, eds. V.T. Covello, W.G.Flamm, J.V. Rodricks, and R.G. Tardiff. New York: Plenum Press, pp. 236-237.

19. Bradbury, op.cit.

20. Ravetz, op.cit.

21. Jurgen Habermas, 1981. The Theory of Communicative Action, vol.1: Reason and the Rationalization of Society (translated by Thomas McCarthy). Boston: Beacon Press. 

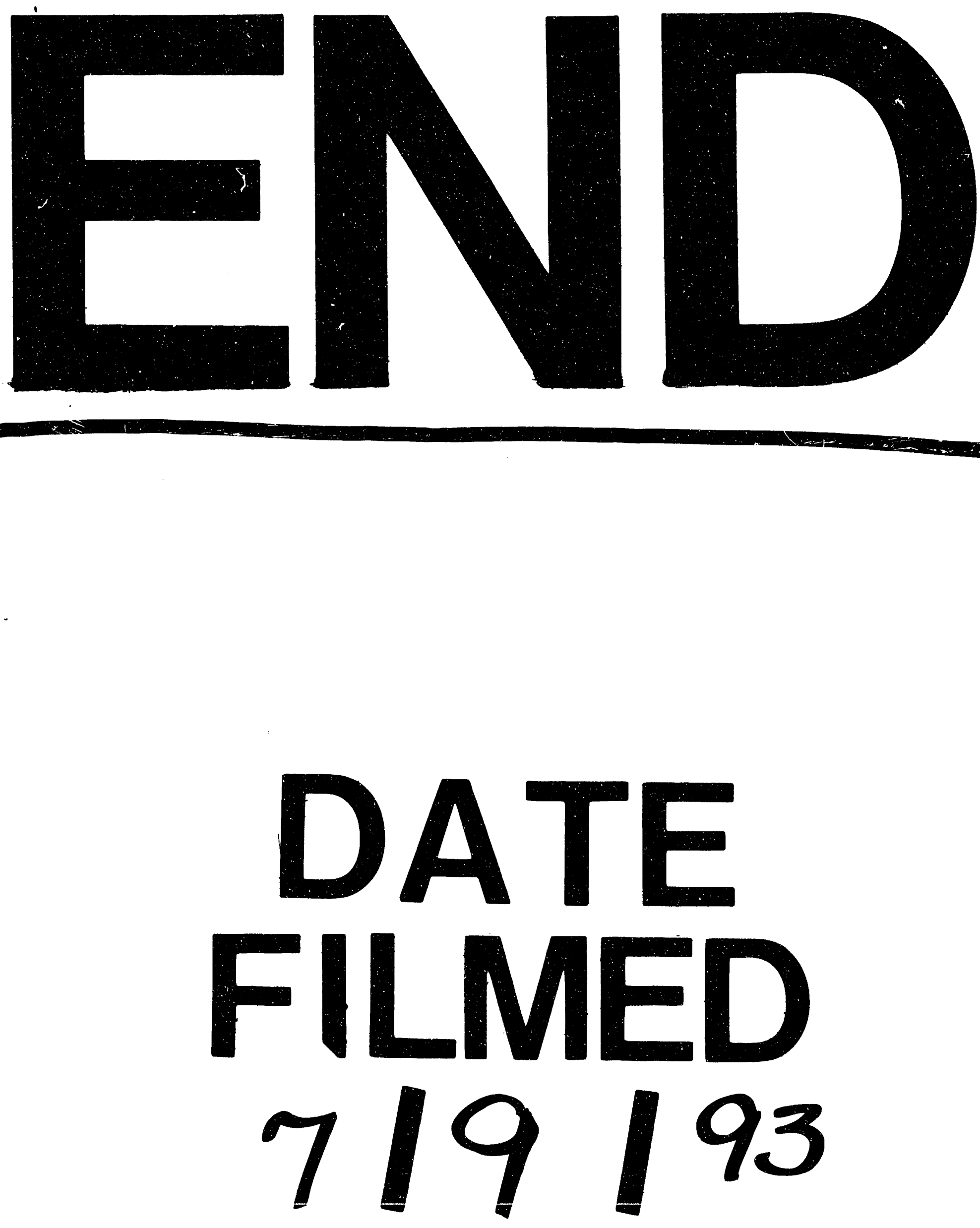
$\doteqdot$ 